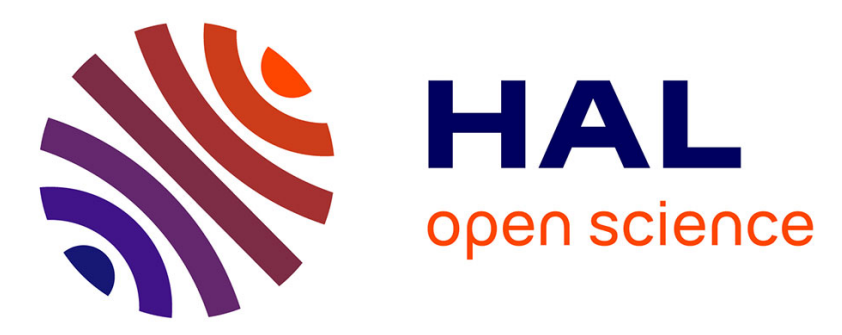

\title{
Interplay between silicate and hydroxide ions during geopolymerization
}

J. Aupoil, J.-B. Champenois, J.-B. d'Espinose de Lacaillerie, A. Poulesquen

\section{To cite this version:}

J. Aupoil, J.-B. Champenois, J.-B. d'Espinose de Lacaillerie, A. Poulesquen. Interplay between silicate and hydroxide ions during geopolymerization. Cement and Concrete Research, 2019, 115, pp.426-432. cea-02339844

\section{HAL Id: cea-02339844 https://hal-cea.archives-ouvertes.fr/cea-02339844}

Submitted on 5 Nov 2019

HAL is a multi-disciplinary open access archive for the deposit and dissemination of scientific research documents, whether they are published or not. The documents may come from teaching and research institutions in France or abroad, or from public or private research centers.
L'archive ouverte pluridisciplinaire HAL, est destinée au dépôt et à la diffusion de documents scientifiques de niveau recherche, publiés ou non, émanant des établissements d'enseignement et de recherche français ou étrangers, des laboratoires publics ou privés. 


\section{Interplay between silicate and hydroxide ions during}

\section{2 geopolymerization}

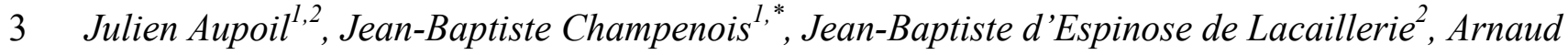

4 Poulesquen ${ }^{1}$

$5 \quad{ }^{1}$ CEA, DEN, DE2D, SEAD, LCBC, F-30207 Bagnols-sur-Cèze, France

$6 \quad{ }^{2}$ Laboratoire de Sciences et Ingénierie de la Matière Molle, UMR CNRS 7615, ESPCI Paris, PSL

7 Research University, 10, rue Vauquelin, 75231 Paris Cedex 05, France

$8 \quad{ }^{*}$ Corresponding author

9 Keywords: silicate, hydroxide, Hammet, alkali, metakaolin, dissolution, condensation, 


\section{Abstract}

13 Two set of activating solutions were prepared with increasing sodium hydroxide content, either

14 containing or not silicates. Their alkalinities, here defined as the ability of solutions to resist

15 changes in $\mathrm{pH}$, were determined and compared by measuring Hammet acidity functions that can

16 be assimilated to extended $\mathrm{pH}$ values. Such Hammet functions in sodium silicate solutions are

17 reported for the first time. The impact of both the alkalinity and the initial Hammett function on

18 the reactivity of metakaolin (MK)-based pastes was assessed using Isothermal Conduction micro-

19 Calorimetry (ICC). It was concluded that the reactivity of MK mixed with sodium hydroxide

20 solution related directly to the Hammett function values whereas in sodium silicate mixes, the

21 alkalinity value was a more pertinent parameter. A mechanism was deduced to clarify the role of

22 hydroxide ions during the geopolymerization, highlighting at the same time the role of silicate

23 species as hydroxide reservoir to nurture the dissolution process. 


\section{1. Introduction}

26 Geopolymers refer to alumino-silicate binders obtained by reaction of a powdered alumino-

27 silicate source, such as dehydroxylated kaolin (metakaolin, MK), with an alkali hydroxide or

28 alkali silicate solution, as described by Davidovits ${ }^{1}$. Schematically, the geopolymerization

29 process can be described by three simplified steps ${ }^{2-5}$. First, MK dissolution in the activating

30 solution provides aluminate and silicate species to the reacting medium. These species then

31 rearrange in solution, to finally polycondense, yielding the 3D network of the hardened

32 geopolymer.

33 Carrying out a detailed mechanistic study of these three steps is a hard task. They occur

34 concurrently and furthermore, no realistic reaction equations can be written to describe any of

35 these steps. However, since most of the expected reaction steps are exothermic, the use of

36 Isothermal Conduction Calorimetry (ICC) was previously reported ${ }^{6-11}$ as being an efficient tool

37 for studying the whole geopolymerization process. Indeed, heat flow and heat profiles recorded

38 during geopolymerization provide qualitative and quantitative information. As an example, the

39 global geopolymerization extent at a given time has been calculated as the ratio of the cumulated

40 heat $\mathrm{Q}(\mathrm{t})$ release to the theoretical total heat at completion, $\mathrm{Q}_{\max }{ }^{6,9,10}$. Hence, in alkali hydroxide

41 activating solutions free from silicates, Zhang et al. ${ }^{9}$ reported an increase of the aforementioned

42 geopolymerization extent with increasing hydroxide ions content.

43 The relationship between activating solution composition and geopolymerization has been the

44 subject of an extensive body of literature. Indeed, such a topic is a key issue for the use of

45 geopolymers and their industrial development. For examples, Rahier et al. ${ }^{12}$ reported that the

46 activating solution modulus, defined as the $\mathrm{SiO}_{2} / \mathrm{Na}_{2} \mathrm{O}$ molar ratio, and the amount of water,

47 defined as the $\mathrm{H}_{2} \mathrm{O} / \mathrm{Na}_{2} \mathrm{O}$ molar ratio, are driving parameters to tune geopolymers stoichiometry 
48 and consistency respectively. At a $\mathrm{Na} / \mathrm{Al}$ molar ratio equal to one, the optimal geopolymer

49 stoichiometry can thus be reached by choosing the appropriate $\mathrm{SiO}_{2} / \mathrm{Na}_{2} \mathrm{O}$ modulus of the

50 activating solution. Later, Duxson et al. ${ }^{13}$ observed an increase in the proportion of unreacted

51 metakaolin and a decrease in the geopolymers density when increasing the silicate content of

52 activating solutions as a consequence of the resulting simultaneous increase of the $\mathrm{SiO}_{2} / \mathrm{Al}_{2} \mathrm{O}_{3}$

53 and $\mathrm{SiO}_{2} / \mathrm{Na}_{2} \mathrm{O}$ molar ratios. Such a result was further rationalized by using Density Functional

54 Theory (DFT)-based Coarse-Grain Monte-Carlo simulations ${ }^{14}$. Similarly, Zhang et al. ${ }^{10}$ reported

55 that an increase in the activating solution modulus, defined as the $\mathrm{SiO}_{2} / \mathrm{Na}_{2} \mathrm{O}$ molar ratio, leads to

56 a decrease in the geopolymerization extent, evaluated by isothermal calorimetry.

57 The use of ${ }^{29}$ Si Nuclear Magnetic Resonance (NMR) clearly exhibited that silicates connectivity 58 in activating solutions increases when increasing the modulus of the solutions ${ }^{15-17}$. Duxson et al. ${ }^{13}$

59 postulated that the higher the silicates connectivity, the lower the silicates species lability.

60 According to these authors, highly connected silicate species would thus hardly rearrange and

61 densify before gelation, leading to the formation of a gel of lesser density and thiner pore size

62 distribution, around metakaolin grains.

64 In most of the aforementioned studies, the activating solutions compositions are described in

65 terms of silicate content, $\mathrm{SiO}_{2} / \mathrm{Na}_{2} \mathrm{O}$ and $\mathrm{H}_{2} \mathrm{O} / \mathrm{Na}_{2} \mathrm{O}$ molar ratios. The influence of the free

66 hydroxide ions content on the geopolymerization reaction is thus not explicit. Although it had

67 already been mentioned by $\mathrm{Xu}$ and $\operatorname{Van}^{\text {Deventer }}{ }^{18}$ and Zhang et $a l^{9}$, to the best of our

68 knowledge, only one scientific paper has been dedicated to that topic. Indeed, by increasing the

69 alkali hydroxide content of activating solutions and using fly ash as an aluminosilicate source,

70 Phair and Van Deventer ${ }^{19}$ reported that a higher activating solution $\mathrm{pH}$ leads to a higher 
71 polycondensation extent. However, $\mathrm{pH}$ reported values seem to be approximate as they are given

72 only in full $\mathrm{pH}$ units ${ }^{19}$. In any case, these three studies ${ }^{9,18,19}$ clearly highlight the importance of

73 hydroxide ions in the geopolymerization process.

74 An effect of solution $\mathrm{pH}$ on aluminosilicates dissolution was indeed evidenced by $\mathrm{Xu}$ and Van

75 Deventer ${ }^{18}$ when studying in diluted suspensions the dissolution of 15 aluminosilicates minerals.

76 They concluded that the extent of dissolution increases for increasing alkali hydroxide solution

77 concentrations. Complementarily, Duxson et $a .^{2}{ }^{2}$ (and references therein) and Granizo et al. ${ }^{20}$

78 amongst others also pinpointed that the dissolution kinetics of aluminosilicate sources was highly

79 dependent on the initial $\mathrm{pH}$ of the solution.

80 However, all the previously mentioned experiments ${ }^{2,18,20}$ were carried out using solutions free

81 from silicates and using high liquid to solid ratio. As such, the role of silicate species and their

82 interplay with hydroxide ions on the dissolution step during geopolymerization have never been

83 investigated. This literature gap may have resulted from the difficulty to assess by standard $\mathrm{pH}-$

84 metry $\mathrm{pH}$ values of solutions containing extremely high amounts of alkali metal ions.

86 In consequence, the present study firstly aims at quantifying the hydroxide content and the

87 alkalinity of two sets of activating solutions. Two sets of solutions of increasing sodium

88 hydroxide content were considered: the first one with a fixed silicate content and the second one

89 devoid of silicate species. Each time, Hammet acidity functions of studied solutions were

90 measured instead of $\mathrm{pH}$, as described in the theoretical part. The corresponding alkalinity ${ }^{21}$,

91 defined here as the ability of a solution to resist to changes in $\mathrm{pH}$, was evaluated for the two sets

92 of solutions. Then, the geopolymers reactivity observed by mixing metakaolin into these

93 solutions was evaluated by using Isothermal Conduction Calorimetry (ICC). Finally, these new 
94 data led us to discuss the role of silicate species and their interplay with hydroxide ions during 95 geopolymerization.

96 


\section{Theoretical basis.}

98 In activating solutions used for geopolymers elaboration, molality of alkali metal ions can range

99 between $1 \mathrm{~mol} / \mathrm{kg}$ of water to $15 \mathrm{~mol} / \mathrm{kg}$ of water. The use of a glass electrode sensitive to

100 oxonium ions $\mathrm{H}_{3} \mathrm{O}^{+}$to assess $\mathrm{pH}$ values is then questionable in such media. First, a high sodium

101 concentration in basic solutions often leads to an alkaline error, since the alkali ion concentration

102 can be typically orders of magnitudes larger than the oxonium ions one. As an example, the alkali

103 ion concentration is $10^{14}$ times higher than the oxonium one in a sodium silicate solution

104 containing $10 \mathrm{~mol} / \mathrm{kg}$ of sodium ions at $\mathrm{pH} \approx 13$. Then, water activity in the studied solution can

105 be far below the value of 0.9 expected in a $3 \mathrm{~mol} / \mathrm{kg}$ potassium chloride electrode filling

106 solution. As a consequence, the ionic flow from the electrode to the studied medium could be

107 strongly impacted, leading to false measurements. Finally, in the specific case of silicate-

108 containing activating solutions, silicate species can interact or even deteriorate the glass electrode

109 surface, leading to additional errors.

110 Consequently, in this work, the measurement of Hammet acidity functions ${ }^{22-24}$ was chosen

111 instead of standard pH-metry to quantify the amount of hydroxide ions in activating solutions.

112 Such a function represents the hydroxide ions ability to deprotonate a weak acid, noted SH in

113 reaction equation 1, added in small amounts in the aqueous medium. It is obviously equivalent to

$114 \mathrm{pH}$ in dilute systems. Upon introduction in the studied medium, again in small amounts, the

115 weak acid SH plays the role of an acid-base indicator whose ionization ratio is measured

116 quantitatively thanks to the intensity shift of its UV-visible spectrum induced by its deprotonation

117 . This concept was initially introduced to measure acidity of extremely acidic solution and later

118 adapted to basic solutions, either in aqueous alkali hydroxide solutions or in non-aqueous 119 solvents ${ }^{25-30}$. 
123 In reaction equation 1 , the coefficient $n=(\mathrm{x}+\mathrm{y}-\mathrm{z})$ accounts for the difference in hydration

124 degrees between reactants and products, according to the model used by Edward ${ }^{29}$. The 125 corresponding equilibrium constant $K$ is then written as:

$126 \quad K=\frac{a_{S-} \cdot a_{H 2 O}^{(n+1)}}{a_{S H} \cdot a_{O H-}}=\frac{K_{a}}{K_{w}} \quad$ (Equation 1)

127 with $a_{i}$ the activity of the species $i$.

128 As reaction equation 1 represents an acid-base reaction between the two couples $\mathrm{SH} / \mathrm{S}^{-}$and

$129 \mathrm{OH}^{-} / \mathrm{H}_{2} \mathrm{O}$, the constant $K$ can also be written as the ratio of the associated ionization constants: $K_{a}$

130 for the acid ionization constant of $\mathrm{SH}$ and $K_{w}$ for the one of water (tabulated by Bandura and

$131 \operatorname{Lvov}^{31}$ and taken as $10^{-14}$ at $\left.25^{\circ} \mathrm{C}\right)$,

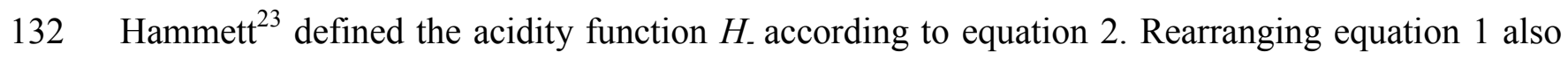

133 provides equation 3 , another definition of the acidity function:

$134 \quad H_{-}=p K_{a}+\log \frac{\left[S^{-}\right]}{[S H]} \quad($ Equation 2)

$135 \quad H_{-}=p K_{w}+\log a_{O H-}-(n+1) \cdot \log a_{H 2 O}+\log \frac{\gamma_{S_{H}}}{\gamma_{S_{-}}} \quad$ (Equation 3$)$

136 With:

$137 p K_{x}$ the cologarithm of constant $K_{x}$,

$138 \quad \frac{[S-]}{[S H]}$ the ionization ratio,

$139[i]$ the concentration of species $i$ in $\mathrm{mol} / \mathrm{kg}$ of water,

140 and $\gamma_{i}$ its activity coefficient defined such as $a_{i}=\gamma_{i \cdot}[i] /[i]^{\circ}$ where $[i]^{\circ}$ is the standard state

141 concentration of species $i$ conventionally set equal to $1 \mathrm{~mol} / \mathrm{kg}$. 
142 The experimental determination of activity coefficients being complex, equation 3 is mainly of

143 theoretical interest, except in the rare cases when good approximations of activity coefficients ${ }^{24,29}$

144 are possible. Nevertheless, equation 3 clearly states that the function $H_{\text {- }}$ takes into account not

145 only the hydroxide ions activity but also their environment through the parameter $n$ and the

146 variables $a_{H 2 O}$ and $\gamma_{S H} / \gamma_{S-}$, respectively related to the ions hydration, the free water content and the

147 ionic force of the solution. In consequence, the acidity function is an appropriate tool to represent

148 the ability of hydroxide ions to react in a solution.

149 If both $\mathrm{S}^{-}$and $\mathrm{SH}$ forms absorb UV-visible light at different wavelengths, the $\left[\mathrm{S}^{-}\right] /[\mathrm{SH}]$

150 ionization ratio can be measured by using spectrocolorimetry. From Beer-Lambert's law, the

151 ionization ratio can be obtained from absorbance measurements for a constant indicator

152 concentration according to (eq. 4).

$153 \quad \frac{\left[S^{-}\right]}{[S H]}=\frac{A-A_{S H}}{A_{S^{-}}-A} \quad($ Equation 4)

$154 \quad$ With

$155 A_{S H}$ the absorbance of a solution where the indicator is fully protonated,

$156 A_{S-}$ the absorbance of a solution where the indicator is fully deprotonated,

$157 A$ the absorbance of any basic solution with intermediate concentration.

158 A set of three solutions is thus needed to determine an ionization ratio. 


\subsection{Activating solutions elaboration}

162 Sodium hydroxide solutions were elaborated with an increasing molality ranging from 3.17 .10

163 to $1.40 .10^{1} \mathrm{~mol} / \mathrm{kg}$ by dissolving sodium hydroxide pellets (AR grade, VWR) in ultrapure water

164 (18.2 M $\Omega)$. A set of alkali silicate solutions was then elaborated by diluting in water a

165 commercial alkali silicate solution $\left(\right.$ Betol $^{\circledR} 52 \mathrm{~T}$, Wöllner: $30.2 \% \mathrm{w} / \mathrm{w} \mathrm{SiO}, 14.7 \% \mathrm{w} / \mathrm{w} \mathrm{Na} \mathrm{N}_{2} \mathrm{O}$ and

$16655.1 \% \mathrm{w} / \mathrm{w} \mathrm{H}_{2} \mathrm{O}$ ) and by adding sodium hydroxide pellets in order to reach increasing sodium

167 ions molality, ranging from 5.46 to $1.40 .10^{1} \mathrm{~mol} / \mathrm{kg}$. All solutions were stirred for at least $4 \mathrm{~h}$

168 and cooled down to $25^{\circ} \mathrm{C}$ prior to any use. Solutions compositions are reported in Tables 1 and 2.

170 Table 1. Composition in molality, water activity and acidity function values of sodium hydroxide

171 solutions together with the molar ratios of the corresponding geopolymers prepared with

172 metakaolin. Acidity functions were taken from literature when indicated ( ${ }^{\mathrm{a}} \operatorname{Rochester}^{32}$,

$173{ }^{\mathrm{b}}$ Schwarzenbach ${ }^{25}$ ).

\begin{tabular}{|c|c|c|c|c|c|}
\hline & \multicolumn{3}{|c|}{ Solutions } & \multicolumn{2}{|c|}{ Geopolymers } \\
\hline & $\begin{array}{c}{[\mathrm{NaOH}]} \\
(\mathrm{mol} / \mathrm{kg})\end{array}$ & $\begin{array}{c}a_{\mathrm{H} 2 \mathrm{O}} \\
( \pm 0.008)\end{array}$ & $\mathrm{H}_{\text {. }}$ & $\mathrm{Na} / \mathrm{Al}$ & $\frac{\mathrm{SiO}_{2}}{\mathrm{Na}_{2} \mathrm{O}}$ \\
\hline $\mathrm{Na1}$ & $3.17 .10^{-2}$ & 1.000 & $12.51 \pm 0.06$ & $3.28 .10^{-3}$ & 733 \\
\hline $\mathrm{Na} 2$ & $1.00 .10^{-1}$ & 1.000 & $12.99 \pm 0.06$ & $1.04 .10^{-2}$ & 232 \\
\hline $\mathrm{Na3}$ & $3.17 .10^{-1}$ & - & $13.42 \pm 0.08$ & $3.28 .10^{-2}$ & 73.2 \\
\hline $\mathrm{Na} 4$ & 1.00 & 0.987 & $14.01^{\mathrm{a}}$ & $1.04 .10^{-1}$ & 23.2 \\
\hline $\mathrm{Na} 5$ & 2.00 & 0.956 & $14.42^{\mathrm{a}}$ & $2.07 .10^{-1}$ & 11.6 \\
\hline $\mathrm{Na} 6$ & 3.01 & 0.917 & $14.72^{\mathrm{a}}$ & $3.11 .10^{-1}$ & 7.73 \\
\hline $\mathrm{Na} 7$ & 5.00 & 0.812 & $15.19^{\mathrm{a}}$ & $5.18 .10^{-1}$ & 4.64 \\
\hline $\mathrm{Na8}$ & 7.00 & 0.692 & $15.62^{\mathrm{a}}$ & $7.25 .10^{-1}$ & 3.32 \\
\hline $\mathrm{Na9}$ & 9.66 & 0.520 & $16.06^{a}$ & 1.00 & 2.41 \\
\hline $\mathrm{Na} 10$ & $1.20 .10^{1}$ & - & $16.47^{b}$ & 1.24 & 1.94 \\
\hline $\mathrm{Na11}$ & $1.40 .10^{1}$ & 0.275 & $16.87^{b}$ & 1.45 & 1.66 \\
\hline
\end{tabular}


177 Table 2. Molality, molar ratio, water activity and acidity function values of sodium silicate

178 solutions. When indicated, the composition of the corresponding geopolymers prepared with

179 metakaolin is also given.

\begin{tabular}{llllllll|cc} 
& \multicolumn{7}{c|}{ Solutions } & \multicolumn{2}{c}{ Geopolymers } \\
\cline { 2 - 10 } & $\begin{array}{c}{[\mathrm{NaOH}]_{\text {added }}} \\
(\mathrm{mol} / \mathrm{kg})\end{array}$ & $\begin{array}{c}{[\mathrm{Na}]_{\text {total }}} \\
(\mathrm{mol} / \mathrm{kg})\end{array}$ & $\begin{array}{c}{[\mathrm{Si}]} \\
(\mathrm{mol} / \mathrm{kg})\end{array}$ & $\frac{\mathrm{SiO}_{2}}{\mathrm{Na}_{2} \mathrm{O}}$ & $\begin{array}{c}\mathrm{a}_{\mathrm{H} 2 \mathrm{O}} \\
( \pm 0.008)\end{array}$ & $\mathrm{H}$. & $\mathrm{Na} / \mathrm{Al}$ & $\frac{\mathrm{SiO}_{2}}{\mathrm{Na}_{2} \mathrm{O}}$ \\
\hline NaS1 & 0 & 5.46 & 5.79 & 2.12 & 0.965 & - & 0.57 & 6.36 \\
NaS2 & $5.26 .10^{-1}$ & 6.01 & 5.80 & 1.93 & 0.952 & $10.82 \pm 1.98$ & 0.62 & 5.79 \\
NaS3 & 1.54 & 7.01 & 5.80 & 1.65 & 0.928 & $11.64 \pm 0.35$ & 0.73 & 4.97 \\
NaS4 & 2.54 & 8.02 & 5.79 & 1.45 & 0.903 & $12.19 \pm 0.14$ & 0.83 & 4.34 \\
NaS5 & 3.53 & 9.00 & 5.80 & 1.29 & - & $12.52 \pm 0.10$ & - & - \\
NaS6 & 4.20 & 9.68 & 5.80 & 1.20 & 0.837 & $12.74 \pm 0.10$ & 1.00 & 3.60 \\
NaS7 & 5.02 & 10.5 & 5.80 & 1.10 & - & $13.10 \pm 0.11$ & - & - \\
NaS8 & 5.52 & 11.0 & 5.80 & 1.05 & 0.767 & $13.25 \pm 0.12$ & 1.14 & 3.16 \\
NaS9 & 6.54 & 12.0 & 5.80 & $9.66 .10^{-1}$ & 0.711 & $13.59 \pm 0.20$ & 1.24 & 2.68 \\
NaS10 & 7.54 & 13.0 & 5.79 & $8.91 .10^{-1}$ & 0.649 & $14.12 \pm 0.53$ & 1.34 & 2.32 \\
NaS11 & 8.54 & 14.0 & 5.80 & $8.27 .10^{-1}$ & - & - & - & - \\
\hline
\end{tabular}

\subsection{Geopolymers preparation}

184 Prior to the cure and any measurements, geopolymer pastes were prepared by mixing $32.05 \mathrm{~g}$ of

185 metakaolin (MK, Metakaolin Argical M1000 from Imerys, characteristics in Table 3) with the

186 appropriate weight of activating solution to obtain a constant (initial water)/MK weight ratio of

187 0.78. Elaborated geopolymers formulations are reported in Tables 1 and 2.

189 Table 3. Physical and chemical characterization of Metakaolin by XRF, laser granulometry and

$190 \mathrm{~N}_{2}$ adsorption-desorption (with BET method) as provided by the supplier.

\begin{tabular}{lcccccc}
\hline Oxides & $\mathrm{SiO}_{2}$ & $\mathrm{Al}_{2} \mathrm{O}_{3}$ & $\mathrm{CaO}$ & $\mathrm{Fe}_{2} \mathrm{O}_{3}$ & $\mathrm{TiO}_{2}$ & $\mathrm{~K}_{2} \mathrm{O}$ \\
\hline Composition \%w/w & 54.40 & 38.40 & 0.10 & 1.27 & 1.60 & 0.62 \\
\hline Granulometry $(\mu \mathrm{m})$ & $\mathrm{d}_{10}$ & 1.8 & $\mathrm{~d}_{50}$ & 10.3 & $\mathrm{~d}_{90}$ & 48.2 \\
\hline surface area $\left(\mathrm{m}^{2} / \mathrm{g}\right)$ & \multicolumn{7}{c}{18} \\
\hline
\end{tabular}




\subsection{Acidity function measurements}

194 Thiazole Yellow G (TYG, also known as Titan Yellow, Sigma-Aldrich) was used in the present

195 study as the weak acid UV-visible-sensitive indicator, chosen to be compatible with the expected

196 acidity function range of the studied solutions. Absorptions of activating solutions containing

197 TYG were measured in the spectral range 300 to $600 \mathrm{~nm}$, with a resolution of $1 \mathrm{~nm}$, in $10-\mathrm{mm}$

198 quartz cells at $25^{\circ} \mathrm{C}$. Absorbance spectra were recorded on a dual-beam spectrophotometer

199 (Genesys 10S UV-Visible, ThermoFischer Scientific), with a xenon flash lamp. Indicator-free

200 activating solutions were used as backgrounds so that only the indicator would contribute to the

201 recorded spectra. For the whole set of solutions, absorbance of indicator-free solutions was

202 checked to be close to zero for wavelength ranging from 400 to $500 \mathrm{~nm}$.

203 The absorbance at $480 \mathrm{~nm}$ has been checked to be proportional to TYG concentration up to

204 approximately $5.10^{-4} \mathrm{~mol} / \mathrm{kg}$ in sodium hydroxide solution, defining the range of validity of the

205 Beer-Lambert law (details are available in supplementary information). Accordingly, the working

206 concentration of TYG in all activating solutions was set to $5.10^{-5} \mathrm{~mol} / \mathrm{kg}$. In sodium hydroxide

207 solutions, absorption bands centered at 405 and $475 \mathrm{~nm}$ have been observed respectively for the

$208 \mathrm{SH}$ form and $\mathrm{S}^{-}$form, with an isobestic point at $433 \mathrm{~nm}$. In sodium silicate solution with

209 increasing amounts of sodium hydroxide, absorption bands were centered at 390 and $480 \mathrm{~nm}$,

210 with an isobestic point at $438 \mathrm{~nm}$. Previous observations from Allain and Xue ${ }^{33}$ in sodium

211 hydroxide solutions support the existence of a simple acid-base equilibrium for TYG as described

212 in Reaction Equation 1. Allain and Xue also reported that TYG has a good chemical resistance to

213 hydroxide ions, and a large dynamic spectral range. In addition, the similar position of the $\mathrm{S}^{-}$

214 form absorption band in both media corroborates the absence of any drastic conformation 
215 modification, at least for the deprotonated form. This meant that silicates did not interact to any

216 significant level with the indicator and that the latter was only involved in a simple acid-base

217 equilibrium. Acidity functions were thus calculated from absorbance at $480 \mathrm{~nm}$.

218 The $p K_{a}$ of TYG has been measured in sodium hydroxide solution following Safavi and

219 Abdollahi's procedure ${ }^{34}$. The $p K_{a}$ value of $12.92 \pm 0.01$ at $25^{\circ} \mathrm{C}$ measured in this work is

220 consistent with the 12.92 value reported by Safavi and Abdollahi. TYG was thus considered as a

221 suitable weak acid indicator for acidity function measurements in study alkali silicate activating

222 solutions.

223 Finally, the working range of TYG was established (see Sup. Inf.) to be comprised between 11.9

224 and 13.9 , corresponding to an $H_{\text {- }}$ range equal to approximately $p K_{a} \pm 1$, which is consistent with

225 the dynamic spectral range found by Safavi and Abdollahi ${ }^{34}$ and Allain and Xue ${ }^{33}$.

\section{3.4. Water activity measurements}

228 Water activity was measured in all considered activating solutions in presence of $5.10^{-5} \mathrm{~mol} / \mathrm{L}$ of

229 TYG. Measurements were done at $25 \pm 2^{\circ} \mathrm{C}$ with a Hygropalm HP23-AW-A analyzer equipped 230 with a HC2-AW water activity probe (Rotronic) and calibrated in temperature and humidity with

231 Rotronic certified humidity standards at $50 \% \mathrm{RH}$ and $80 \% \mathrm{RH}$. After an equilibration time of

2325 min, water activity was measured with a precision of \pm 0.008 . Water activity measurement

233 relies on Equilibrium Relative Humidity measurement (ERH, in \%), when the atmosphere in the

234 sample holder is at equilibrium with the solution $\left(a_{H 2 O}=\mathrm{ERH} / 100\right)$. 
$237{ }^{29} \mathrm{Si}$ NMR measurements were performed on silicate activating solutions to investigate the silicate 238 connectivity. Spectra were recorded at a Larmor frequency of $99.36 \mathrm{MHz}$ (at $25 \pm 2^{\circ} \mathrm{C}$ ) in 239 zirconia rotors using a Bruker Avance spectrometer and a 7-mm commercial Bruker MAS probe 240 but without spinning. 1600 transients were acquired using a single $90^{\circ}$ pulse of $6.4 \mu$ s and a 241 recycle time of $10 \mathrm{~s}$. The recycle time was verified to be long enough by increasing it ten folds 242 and confirming that intensities did not vary. Spectra were referenced externally to 243 tetramethylsilane (TMS). The proportions of the different types of silicon centers were obtained

244 by the integrated intensities of their resonances using the software Dmfit developed by D. 245 Massiot et al. $^{35}$. The different silicon centers were designated according to Engelhardt's

246 nomenclature ${ }^{15}$. Each center is designated as $Q$ since silicon atoms are quadri-coordinated to 247 oxygen. A superscript $Q^{n}(0 \leq n \leq 4)$ indicates the number of siloxo bonds (Si-O-Si), without 248 considering the protonation degree of non-bridging oxygens. For example $Q^{0}$ designates a single

249 silicate, $Q^{l}$ a silicate with one neighbor silicate (end-chain or in a dimer) and so on. When 250 present, subscript $c$ indicates that silicates are part of a three-membered ring. These $Q^{2}$ 251 resonances are detected at slightly different frequencies compared to the ones of $Q^{2}$ groups in 252 chains or larger rings due to their more constraint geometry.

253

254 3.6. Isothermal Conduction Calorimetry

255 Approximately $5 \mathrm{~g}$ of each elaborated geopolymer has been then introduced in a sealed ampoule 256 to assess its reactivity by using Isothermal Conduction Calorimetry at $25^{\circ} \mathrm{C}$ on a TAM Air 257 microcalorimeter. Water was used as the reference, to compensate for possible external 258 temperature disturbances. Due to the external mixing procedure, a parasitic heat flow signal 259 associated to the introduction of sample interfered with the initial reaction signal. The 
260 equilibration time of this interference amounted to about $1.5 \mathrm{~h}$, measured on an inert sample

261 (available in supplementary information). Normalized heat flow release during 262 geopolymerization, expressed in $\mathrm{mW} / \mathrm{g}$ of paste, was recorded as a function of time during $90 \mathrm{~h}$.

263 Cumulative heat releases were obtained by integrating heat flow profiles after the end of the 264 introduction peak here defined as when the heat flow drops to a value of $8 \mathrm{~mW} / \mathrm{g}$ to minimize 265 the contribution of the introduction peak. 


\section{Results}

\section{4.1. Acidity function $H_{\text {- }}$ and alkalinity evaluation.}

270 Acidity function values of were measured for the first time in sodium silicate solutions and 271 compared to the ones of pure sodium hydroxide solutions. Results are plotted as a function of 272 added sodium hydroxide molality in Figure 1.

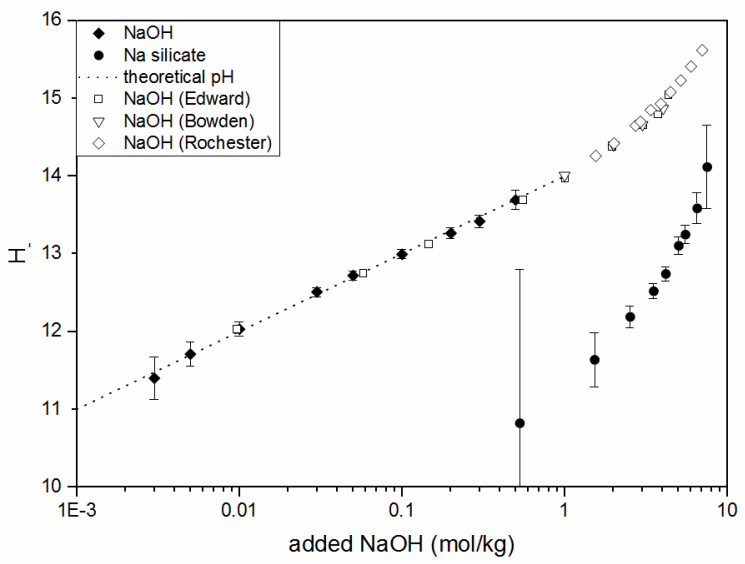

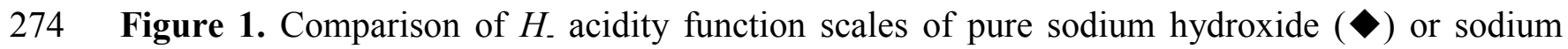

275 silicate solutions $(\mathrm{O})$ (with $[\mathrm{Si}]=5.8 \mathrm{~mol} / \mathrm{kg}$ ) as a function of added sodium hydroxide 276 molality. Literature data from $\operatorname{Edward}^{29}(\square), \operatorname{Bowden}^{30}(\nabla)$ and $\operatorname{Rochester}^{32}(\diamond)$ were used for 277 highly concentrated sodium hydroxide solutions. The dash line represents calculated theoretical

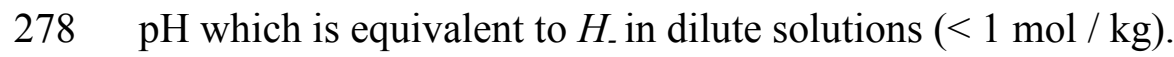

280 In a sodium hydroxide solution free from silicate, an addition of approximately $0.97 \mathrm{~mol} / \mathrm{kg}$

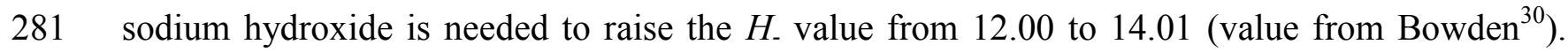

282 Within this concentration range and in the specific case of pure sodium hydroxide solutions, $H_{-}$is 283 fairly equivalent to theoretical $\mathrm{pH}$ calculated by $p K_{w}+\log \left[\mathrm{OH}^{-}\right]$(with a mean deviation of 0.03 ) 284 and our measured values were consistent with previously reported data ${ }^{29}$. Above $1 \mathrm{~mol} / \mathrm{kg}, H_{\text {- }}$ 
285 values are out of the working range of TYG, and cannot be measured with this indicator. For 286 sodium hydroxide addition higher than $1 \mathrm{~mol} / \mathrm{kg}$, the deviation of the $H_{-}$function from the

287 linearity is illustrated by plotting data from the literature ${ }^{29,30,32}$. Such a deviation is mainly due to 288 a sharp decrease in water activity as mentioned by Edward ${ }^{29}$, which was measured for reference 289 and reported in Tables 1 and 2.

290 In a silicate-containing solution, an addition of approximately $4.0 \mathrm{~mol} / \mathrm{kg}$ sodium hydroxide was

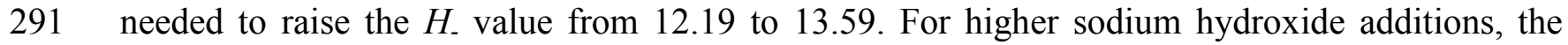

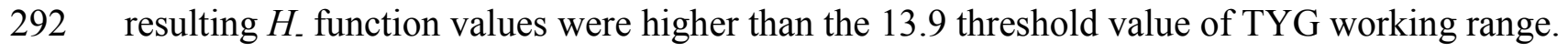

293 This set of data remarkably highlights the alkalinity difference between sodium hydroxide and 294 sodium silicate solutions. Alkalinity is here defined as the ability of a solution to resist changes in $295 \mathrm{pH}$ in a given $\mathrm{pH}$ range. Using this working definition, the alkalinity of both sets of studied 296 solutions was calculated as the amount of hydroxide ions that has to be added to the solution to

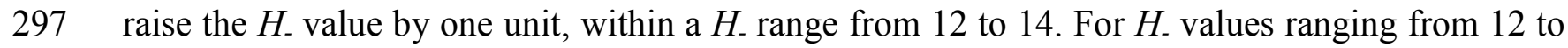
298 14, the alkalinity values were found to be 0.48 mole added hydroxide per $H$. unit for a silicate299 free solution and 2.86 for a solution containing $5.80 \mathrm{~mol} / \mathrm{kg}$ of silicate,. Within the same acidity 300 function range, the alkalinity of the silicate-containing solution is thus more than 5 times higher 301 than the one of the silicate-free solution.

\section{4.2. Silicate connectivity}

304 Liquid state ${ }^{29} \mathrm{Si}$ NMR spectra of some sodium silicate solutions investigated herein are reported 305 on Figure 2. Several types of silicate species were present. The most deshielded are $Q^{0}$ silicates $(\delta$ $306=-71.45 \mathrm{ppm}$ in NaS1$)$ and then $Q^{I}(\delta=-79.45 \mathrm{ppm}$ in NaS1 $), Q_{c}^{2}\left(\delta=-81.36 \mathrm{ppm}\right.$ in NaS1) $Q^{2}$ $307\left(\delta=-87.47 \mathrm{ppm}\right.$ in NaS1), $Q_{c}^{3}\left(\delta=-89.55 \mathrm{ppm}\right.$ in NaS1), $Q^{3}\left(\mathrm{i}=-95.52 \mathrm{ppm}\right.$ in NaS1). A $Q^{4}(\delta=$ 
$308-105.4 \mathrm{ppm}$ ) resonance was only observed in $\mathrm{NaS} 1$. For indication, the ${ }^{29} \mathrm{Si}$ resonances are known 309 to shift to higher frequencies (higher deshielding) when increasing the alkali hydroxide content.

310 This well-known fact is due both to the deprotonation of silanol groups ( $\mathrm{Si}-\mathrm{OH})$ and to the

311 formation of ion pairs ( $\left.\mathrm{Si}-\mathrm{O}^{-+} \mathrm{Na}\right)$ according to Kinrade and Swaddle ${ }^{17}$. As expected, when the

312 alkali hydroxide content is increased, the peak intensities decreased for highly connected silicates

313 and increases for poorly connected silicates, reflecting the general decondensation of silica

314 oligomers with $\mathrm{pH}$. Poorly resolved $Q^{2}$ and $Q_{c}^{3}$ contributions were decomposed by fitting spectra

315 with gausso-lorentzian lineshapes using the freeware Dmfit ${ }^{35}$. The average connectivity of Si

316 centers was calculated from the spectral decomposition of ${ }^{29} \mathrm{Si}$ NMR measurements:

$$
c=\frac{\sum_{n} n \cdot Q^{n}}{\sum_{n} Q^{n}}
$$

317 with $Q^{n}$ the proportion of each silicate population (\%). 


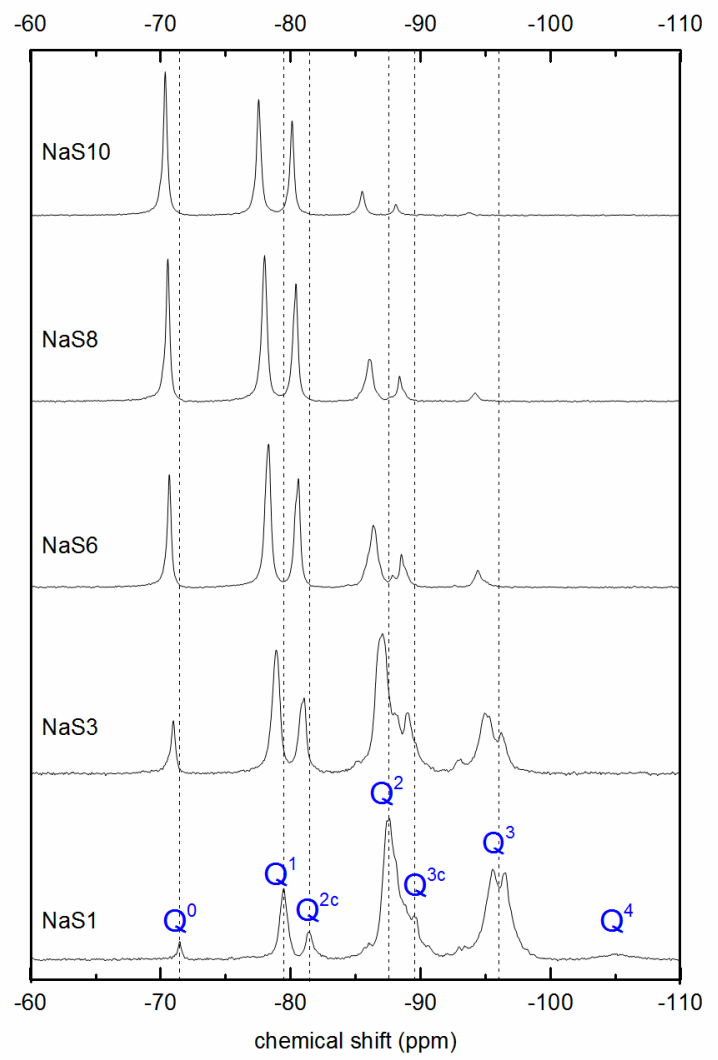

320 Figure 2. Liquid state ${ }^{29} \mathrm{Si}$ NMR spectra of some sodium silicate solutions investigated herein

321 with varying sodium hydroxide contents. Peaks are named after Engelhardt notation ${ }^{15}$.

323 On Figure 3, the average silicate connectivity is plotted as a function of acidity function values in

324 silicate solutions. In the working range of TYG (dash lines), the average connectivity decreases

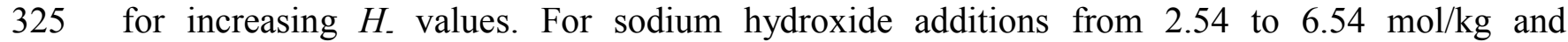

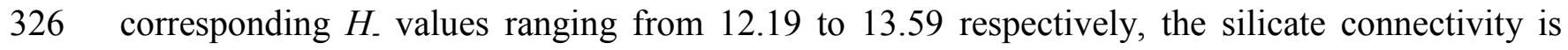

327 divided by almost 2. It demonstrates the decondensation of silicates species when sodium

328 hydroxide is added to solutions, as already reported by many authors. ${ }^{15-17}$ Decondensation

329 consists in the hydrolysis of siloxo bonds Si-O-Si. Such an effect, highlighted by Svensson et 
$330 a l .^{16}$, can be seen as the transfer of hydroxide ions from the solution onto silicates in the form of

331 Si-OH groups. This leads to the consumption of initially added hydroxide ions, as illustrated by

332 Reaction Equation 2:

$$
(\equiv \mathrm{Si}-\mathrm{O}-\mathrm{Si} \equiv)_{\mathrm{aq}}+\mathrm{OH}^{-} \leftrightarrow \equiv \mathrm{Si}-\mathrm{OH}+{ }^{-} \mathrm{O}-\mathrm{Si} \equiv \quad \text { (Reaction Equation 2) }
$$

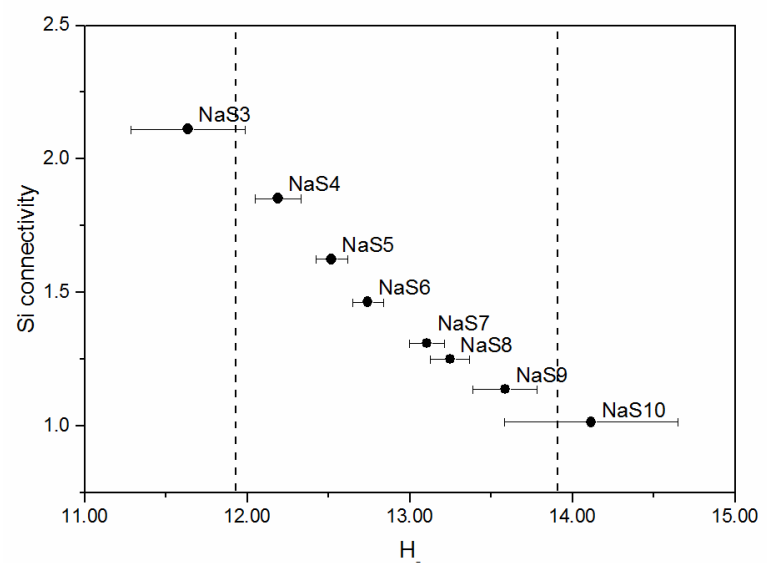

335 Figure 3. Evolution of the average connectivity of silicate oligomers in sodium silicate activating

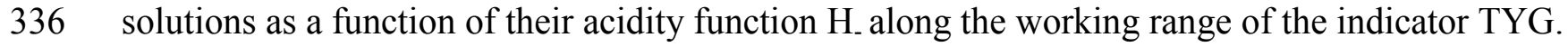

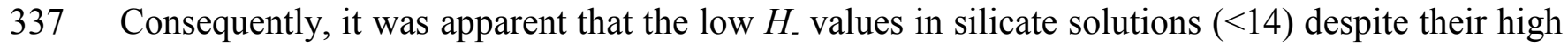

338 sodium hydroxide contents, up to $7.5 \mathrm{~mol} / \mathrm{kg}$, were due to the presence of silicate species in

339 solutions and to the associated reaction described above. The presence of silicate was thus

340 responsible for the alkalinity measured in silicate-containing solutions.

\section{$342 \quad$ 4.3. Isothermal Conduction Calorimetry measurements}

343 Metakaolin-based pastes were then prepared with the solutions under investigation. For all the

344 studied compositions, cumulative heat profiles could be described as a succession of two stages ${ }^{6-}$

$345{ }^{11}$ (Figure 4). The sharp heat release at the early beginning of the reaction can be ascribed mainly 346 to the metakaolin dissolution. But this contribution often overlapped second stage of the 
347 geopolymerization, namely the alumino-silicate polycondensation. After a variable duration, the

348 heat release stabilized as the reaction slowed down. The heat value at the plateau (Figure 4 and 5)

349 could then be assumed to be approximately proportional to the extent of geopolymerization. ${ }^{6,9,10}$
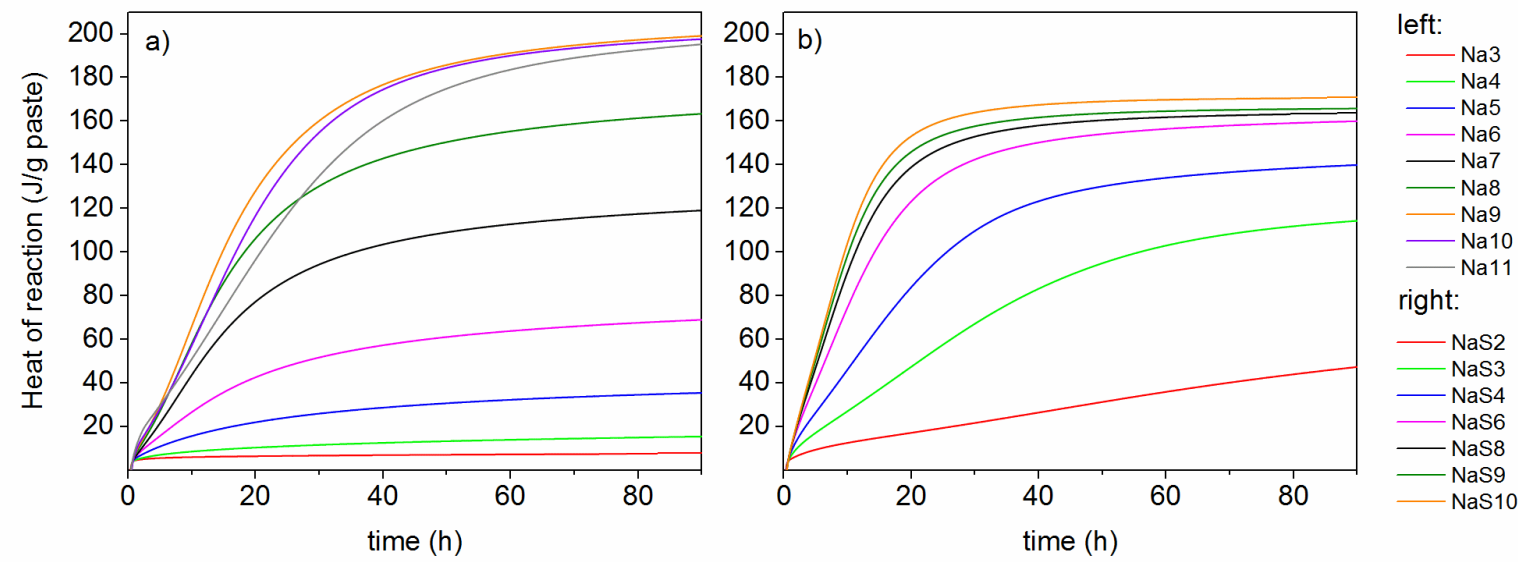

350

351 Figure 4. Total heat release of geopolymer pastes prepared from a) sodium hydroxide solutions 352 and b) sodium silicate solutions, with varying $\mathrm{NaOH}$ content, measured by ICC. 
355 The final heat value was plotted as a function of total sodium ions content. In the same figure, the

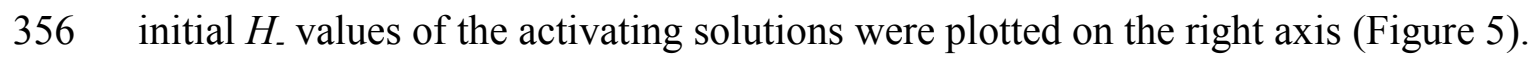

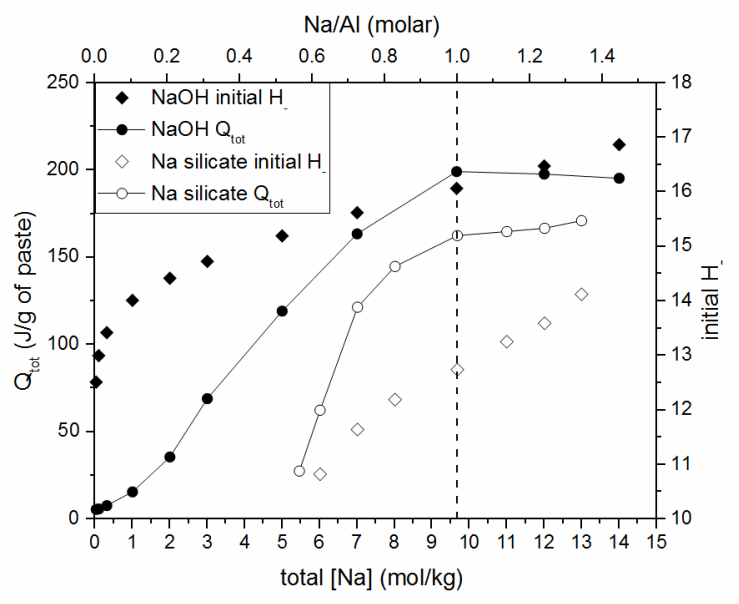

359 Figure 5. Comparison of the final heat released at $90 \mathrm{~h}$ of MK-based geopolymers measured by

360 Isothermal Conduction Calorimetry $(\bullet)$ with the initial $H_{\text {- function }}(\bullet)$ in either pure sodium

361 hydroxide solutions (solid symbols) or sodium silicate solutions (empty symbols) as a function of

362 total sodium molality.

363 For both sets of experiments, i. e. with or without silicate in the activating solution, increasing the

364 total sodium ions molality by adding sodium hydroxide up to $9.66 \mathrm{~mol} / \mathrm{kg}$ led to an increase in

365 the heat of geopolymerization. Above this $9.66 \mathrm{~mol} / \mathrm{kg}$ value, the heat released during

366 geopolymerization remained constant. It has to be noted that this molality value corresponded to

367 a Na/Al ratio equal to 1 , described in literature ${ }^{36}$ as the optimal geopolymer stoichiometry due to

368 the charge balance between $\mathrm{Na}^{+}$ions and $\mathrm{AlO}_{4}{ }^{-}$units. At this point, the initial $H_{-}$values of

369 activating solutions amount to 12.7 and 16.1 respectively in silicate-containing and silicate-free

370 activating solution. At this $9.66 \mathrm{~mol} / \mathrm{kg}$ sodium molality, $(\mathrm{Na} / \mathrm{Al}=1)$, the $H_{\text {- }}$ value in the 
371 silicate-containing solution was thus more than 3 units lower than in the silicate-free one. Despite

372 this huge difference, and for the same total sodium ions concentration, geopolymerization extents

373 were rather close in both systems.

375 While, it was predictable that the composition of the reactants with respects of the stoichiometry 376 of the final geopolymer product $(\mathrm{Na} / \mathrm{Al}=1)$ piloted the possibility or not to reach completion, 377 and that this explains the dependence on the sodium content of the activating solution, it 378 remained surprising at first glance that the silicate containing activating solutions, despite their

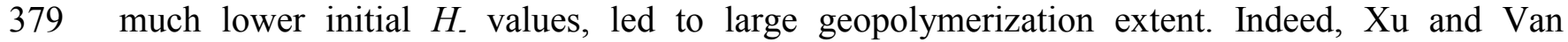

380 Deventer ${ }^{18}$ have demonstrated among others that increasing the $\mathrm{pH}$ value of the dissolution media 381 enhances alumino-silicate minerals dissolution and one might expect consequently a much lower 382 extent of reaction for metakaolin in silicate solutions than in pure $\mathrm{NaOH}$ ones. The benefits in 383 adding silicate species in activating solutions to enhance the geopolymerization process have 384 been previously mainly attributed to their role on condensation reactions, since silicate species 385 are available from the beginning of geopolymerization according to Duxson et al. ${ }^{13}$. Moreover,

386 Phair and Van Deventer ${ }^{19}$ have shown that increasing the alkali content leads to less condensed 387 and more labile silicate species. A more porous and less dense geopolymer would thus be yielded 388 at lower modulus $\mathrm{SiO}_{2} / \mathrm{Na}_{2} \mathrm{O}^{13,19}$.

389 However, the data presented here suggest another important role for the silicate species. When 390 using a silicate-free solution as the activating solution, hydroxide ions are consumed by 391 metakaolin dissolution, as described by Reaction Equation 3 as already reported by Xu and Van 392 Deventer ${ }^{18}$. Due to the low alkalinity of sodium hydroxide solutions, meaning, as defined in the 
393 introduction, that they do not oppose $\mathrm{pH}$ changes, the $\mathrm{pH}$ necessarily drops during reaction and,

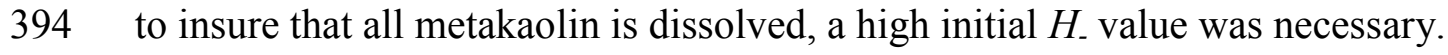

$$
(\equiv \mathrm{Si}-\mathrm{O}-(\mathrm{Si}, \mathrm{Al}) \equiv)_{\text {metakaolin }}+\mathrm{OH}^{-} \leftrightarrow(\equiv \mathrm{Si}-\mathrm{OH})_{\text {metakaolin }}+\left({ }^{-} \mathrm{O}-(\mathrm{Si}, \mathrm{Al}) \equiv\right)_{\mathrm{aq}}(\text { Reaction Equation } 3)
$$

398 At the opposite, when a silicate-containing solution was used to activate metakaolin and for a same $\mathrm{Na} / \mathrm{Al}$ ratio (typically 1 ), the $H_{\text {- }}$ initial values were lower but a similar level of 400 geopolymerization was reached. Obviously, the lower acidity function $H_{\text {- values were }}$ 401 compensated by the strong alkalinity resulting from the presence of silicate species. While 402 hydroxide ions are also consumed by the metakaolin dissolution which releases aluminate and 403 silicate species in solution, following Equation 3, the hydroxide are removed from solution but 404 the concomitant release of silicate and aluminate species in solution favors condensation of pre405 existing alumino-silicate species, thus releasing further hydroxide ions or water molecules in 406 solution (Reaction Equations 4 and 5). Those freshly released hydroxide ions are then available to 407 react in turn at the metakaolin surface, releasing more and more silicate and aluminate species 408 which can feed the condensation. As a result, a chain reaction can be established thanks to this 409 circular mechanism that nurtures the dissolution process. This phenomenon is enhanced in 410 silicate-containing solutions due to their alkalinity. In other terms, the initial silicate in solution 411 act as a reservoir of hydroxide compensating the effect of the $H_{\text {- values which are initially lower }}$ 412 than in silicate-free solutions but remain probabbly somewhat constant during the dissolution.

$$
\left.(\equiv \mathrm{Si}-\mathrm{OH})_{\mathrm{aq}}+\left({ }^{-} \mathrm{O}-(\mathrm{Si}, \mathrm{Al}) \equiv\right)_{\mathrm{aq}} \leftrightarrow(\equiv \mathrm{Si}-\mathrm{O}-(\mathrm{Si}, \mathrm{Al}) \equiv)_{\mathrm{aq}}+\mathrm{OH}^{-} \quad \text { (Reaction Equation } 4\right)
$$

$414 \quad(\equiv \mathrm{Si}-\mathrm{OH})_{\mathrm{aq}}+(\mathrm{HO}-(\mathrm{Si}, \mathrm{Al}) \equiv)_{\mathrm{aq}} \leftrightarrow(\equiv \mathrm{Si}-\mathrm{O}-(\mathrm{Si}, \mathrm{Al}) \equiv)_{\mathrm{aq}}+\mathrm{H}_{2} \mathrm{O} \quad$ (Reaction Equation 5) 
415 It is understood, as shown in Reaction Equation 5, that condensation can also possibly release

416 water molecules. Nevertheless, it has been shown that $\mathrm{Si}(\mathrm{OH})_{2} \mathrm{O}_{2}{ }^{2-}$ and $\mathrm{Si}(\mathrm{OH})_{3} \mathrm{O}^{-}$are the

417 predominant forms of silicate monomers in activating solutions for geopolymers with respective

$418 p K_{a}$ 's of 12.6 and $15.7^{37}$. Besides, silicates $p K_{a}$ 's decrease with their connectivity ${ }^{37}$. In

419 consequence, the level of silicate deprotonation should thus be significant in the present

420 activating solutions. The release of hydroxide ions rather than water molecules would thus be

421 favored.

\section{5. Conclusion}

423 Comparing initial $H$. values at identical $\mathrm{Na}$ content in sodium hydroxide and sodium silicate

424 allowed assessing for the first time the role of activating solution alkalinity in the

425 geopolymerization process, where the alkalinity is defined as the ability of activating solutions to

426 resist to $H$. changes. As a consequence, the role of silicate species in the geopolymerization

427 process is indirectly highlighted, since the presence of silicate species is responsible for

428 activating solutions alkalinity.

429 The basicity of geopolymer activating solutions was investigated by UV-Visible spectroscopy

430 using Thiazole Yellow G as a weakly acidic indicator to calculate their Hammett acidity function

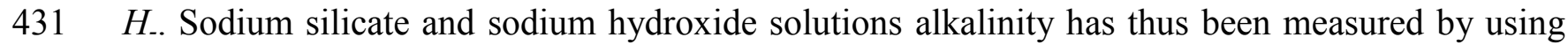

432 this methodology. The present results on sodium hydroxide solutions were consistent with

433 previous results from Safavi ${ }^{34}$, validating the implemented methodology. For the first time to our

434 knowledge, this technique was applied to alkali silicate solutions. Quantitative data on the

435 basicity of geopolymer activating solutions were obtained, without using $\mathrm{pH}$-metry. Thiazole

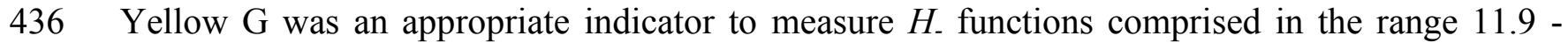

437 13.9, which is ideally suitable for concentrated alkali silicate activating solutions. 
438 The variation of acidity function was found low in silicate-based solutions when compared to 439 silicate-free solutions, for similar sodium hydroxide additions. This alkalinity difference, defined 440 as the ability of studied solutions to resist changes in $\mathrm{pH}$, is due to the condensation and

441 decondensation of silicate species in the medium, as evidenced by using liquid state ${ }^{29} \mathrm{Si}$ NMR. 442

443 The reactivity of metakaolin-based geopolymers elaborated with previously investigated 444 activating solutions was studied by using Isothermal Conduction Calorimetry. Taking into

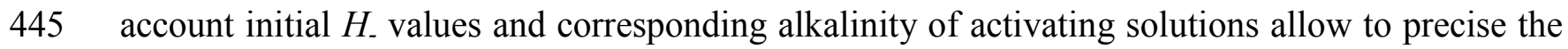
446 respective role of hydroxide ions and silicate species in the geopolymerization process. After 447 metakaolin dissolution initiation by hydroxide ions, the "hydroxide reservoir" on silicates can 448 gradually release hydroxide ions into the solution during the subsequent condensation of 449 alumino-silicate species. Those freshly released hydroxide ions would then be able to dissolve at 450 their turn additional amounts of metakaolin. This would generate a chain reaction and a self451 sustained circular mechanism. This high alkalinity explain why silicate solutions of low initial $H_{\text {- }}$ 452 values lead to a similar extent of geopolymerisation compared to a silicate-free solution

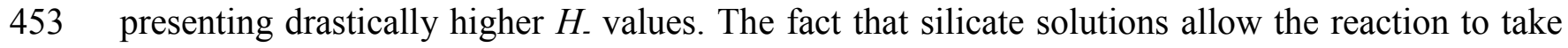
454 place at lower hydroxide concentrations is probably crucial in directing the reaction path towards 455 geopolymers rather than zeolites.

456 Experimental work has to be enriched to further validate this proposition, especially by 457 measuring acidity functions during the course of geopolymerization. It must be understood 458 however that only discontinuous measurements would be possible with this technique, after 459 extracting the solution from the geopolymer paste at different times, since translucent media are 460 required for absorption based methods. 
462 6. Conflicts of interest

463

464 There are no conflicts to declare 


\section{Reference section}

466 (1) Davidovits, J. GEOPOLYMERS - Inorganic polymeric new materials. J. Therm. Anal. 1991, 37 (8), 16331656.

(2) Duxson, P.; Fernandez-Jimenez, A.; Provis, J. L.; Lukey, G. C.; Palomo, A.; van Deventer, J. S. J. Geopolymer technology: the current state of the art. J. Mater. Sci. 2007, 42 (9), 2917-2933.

(3) Provis, J. L.; Duxson, P.; van Deventer, J. S. J.; Lukey, G. C. The role of mathematical modelling and gel chemistry in advancing geopolymer technology. Chem. Eng. Res. Des. 2005, 83 (A7), 853-860.

(4) Fernandez-Jimenez, A.; Palomo, A.; Criado, M. Microstructure development of alkali-activated fly ash cement: a descriptive model. Cem. Concr. Res. 2005, 35 (6), 1204-1209.

(5) Khale, D.; Chaudhary, R. Mechanism of geopolymerization and factors influencing its development: a review. J. Mater. Sci. 2007, 42 (3), 729-746.

(6) Buchwald, A.; Tatarin, R.; Stephan, D. Reaction progress of alkaline-activated metakaolin-ground granulated blast furnace slag blends. J. Mater. Sci. 2009, 44 (20), 5609-5617.

(7) Granizo, M. L.; Blanco, M. T. Alkaline activation of metakaolin - An isothermal conduction calorimetry study. J. Therm. Anal. Calorim. 1998, 52 (3), 957-965.

(8) Yao, X.; Zhang, Z. H.; Zhu, H. J.; Chen, Y. Geopolymerization process of alkali-metakaolinite characterized by isothermal calorimetry. Thermochim. Acta 2009, 493 (1-2), 49-54.

(9) Zhang, Z. H.; Wang, H.; Provis, J. L.; Bullen, F.; Reid, A.; Zhu, Y. C. Quantitative kinetic and structural analysis of geopolymers. Part 1 . The activation of metakaolin with sodium hydroxide. Thermochim. Acta 2012, 539, 23-33.

(10) Zhang, Z. H.; Provis, J. L.; Wang, H.; Bullen, F.; Reid, A. Quantitative kinetic and structural analysis of geopolymers. Part 2. Thermodynamics of sodium silicate activation of metakaolin. Thermochim. Acta 2013, 565, 163-171.

(11) Sun, Z. Q.; Vollpracht, A. Isothermal calorimetry and in-situ XRD study of the $\mathrm{NaOH}$ activated fly ash, metakaolin and slag. Cem. Concr. Res. 2018, 103, 110-122. glasses .3. Influence of the composition of the silicate solution on production, structure and properties. J. Mater. Sci. 1997, 32 (9), 2237-2247.

(13) Duxson, P.; Provis, J. L.; Lukey, G. C.; Mallicoat, S. W.; Kriven, W. M.; van Deventer, J. S. J. Understanding the relationship between geopolymer composition, microstructure and mechanical properties. Colloid Surf. A-Physicochem. Eng. Asp. 2005, 269 (1-3), 47-58.

(14) White, C. E.; Provis, J. L.; Proffen, T.; van Deventer, J. S. J. Molecular mechanisms responsible for the structural changes occurring during geopolymerization: Multiscale simulation. Aiche J. 2012, 58 (7), 2241-2253.

(15) Engelhardt, G.; Zeigan, D.; Jancke, H.; Hoebbel, D.; Wieker, W. ${ }^{29}$ Si NMR-Spectroscopy of Silicate Solutions .II. Dependence of Structure of Silicate Anions in Water Solutions from Na:Si Ratio. Z. Anorg. Allg. Chem. 1975, 418 (1), 17-28.

(16) Svensson, I. L.; Sjoberg, S.; Ohman, L. O. Polysilicate Equilibria in Concentrated Sodium Silicate Solutions. J. Chem. Soc., Faraday Trans. 1 1986, 82, 3635-3646.

(17) Kinrade, S. D.; Swaddle, T. W. Silicon-29 NMR Studies of Aqueous Silicate Solutions .1. Chemical Shifts and Equilibria. Inorg. Chem. 1988, 27 (23), 4253-4259.

508 (19) Phair, J. W.; Van Deventer, J. S. J. Effect of the silicate activator pH on the microstructural 509 characteristics of waste-based geopolymers. Int. J. Miner. Process. 2002, 66 (1-4), 121-143. 
(20) Granizo, N.; Palomo, A.; Fernandez-Jimenez, A. Effect of temperature and alkaline concentration on metakaolin leaching kinetics. Ceram. Int. 2014, 40 (7), 8975-8985.

512 (21) Drever, J. I. The Geochemistry of Natural Water. Pearson Education Canada: 1988.

513 (22) Flexser, L. A.; Hammett, L. P.; Dingwall, A. The Determination of Ionization by Ultraviolet 514 Spectrophotometry: Its Validity and its Application to the Measurement of the Strength of Very Weak Bases. J. Am. Chem. Soc. 1935, 57 (11), 2103-2115.

(23) Hammett, L. P.; Deyrup, A. J. A SERIES OF SIMPLE BASIC INDICATORS. I. THE ACIDITY FUNCTIONS OF MIXTURES OF SULFURIC AND PERCHLORIC ACIDS WITH WATER. J. Am. Chem. Soc. 1932, 54 (7), 27212739.

(24) Hammett, L. P. THE THEORY OF ACIDITY. J. Am. Chem. Soc. 1928, 50 (10), 2666-2673.

(25) Schwarzenbach, G.; Sulzberger, R. Über die Alkalinität starker Lösungen der Alkalihydroxyde. Helv. Chim. Acta 1944, 27 (1), 348-362.

(26) Paul, M. A.; Long, F. A. H AND RELATED INDICATOR ACIDITY FUNCTIONS. Chem. Rev. 1957, 57 (1), 1-45.

(27) Stewart, R.; Odonnell, J. P. STRONGLY BASIC SYSTEMS .III. H. FUNCTION FOR VARIOUS SOLVENT SYSTEMS. Can. J. Chem.-Rev. Can. Chim. 1964, 42 (7), 1681-1693.

(28) Rochester, C. H. Correlation of Acidity Functions with Equilibria of p-Nitroaniline in Aqueous Sodium Hydroxide Solution. Trans. Faraday Soc. 1963, 59 (492), 2820-2825.

(29) Edward, J. T.; Wang, I. C. IONIZATION OF ORGANIC COMPOUNDS .II. THIOACETAMIDE IN AQUEOUS SODIUM HYDROXIDE. THE H. ACIDITY FUNCTION. Can. J. Chem.-Rev. Can. Chim. 1962, 40 (3), 399-407.

(30) Bowden, K. ACIDITY FUNCTIONS FOR STRONGLY BASIC SOLUTIONS. Chem. Rev. 1966, 66 (2), 119131.

(31) Bandura, A. V.; Lvov, S. N. The ionization constant of water over wide ranges of temperature and density. J. Phys. Chem. Ref. Data 2006, 35 (1), 15-30.

(32) Rochester, C. H. Correlation of Reaction Rates with Acidity Functions in Strongly Basic Media .Part 2. Reaction of 2,4-Dinitroaniline With Aqueous Sodium Hydroxide. Trans. Faraday Soc. 1963, 59 (492), 2826-2837.

(33) Allain, L. R.; Xue, Z. L. Optical sensors for the determination of concentrated hydroxide. Anal. Chem. 2000, 72 (5), 1078-1083.

(34) Safavi, A.; Abdollahi, H. Optical sensor for high pH values. Anal. Chim. Acta 1998, 367 (1-3), 167-173. (35) Massiot, D.; Fayon, F.; Capron, M.; King, I.; Calvé, S. L.; Alonso, B.; Durand, J. O.; Bujoli, B.; Gan, Z.; Hoatson, G. Modelling one- and two-dimensional solid-state NMR spectra. Magn. Reson. Chem. 2002, 40 (1), 70-76.

(36) Rahier, H.; VanMele, B.; Biesemans, M.; Wastiels, J.; Wu, X. Low-temperature synthesized aluminosilicate glasses .1. Low-temperature reaction stoichiometry and structure of a model compound. J. Mater. Sci. 1996, 31 (1), 71-79.

(37) Sefcik, J.; McCormick, A. V. Thermochemistry of aqueous silicate solution precursors to ceramics. Aiche J. 1997, 43 (11), 2773-2784. 